九州大学学術情報リポジトリ

Kyushu University Institutional Repository

\title{
The Effects of Noise and Vibration Generated by Mechanized Equipment in Laying Hen Houses on Productivity
}

Oh, Taek-Keun

Biotron Application Center, Kyushu University

Lee, Seung-Joo

Department of Biosystem Machinery Engineering, Chungnam National University

Chang, Dong-I l

Department of Biosystem Machinery Engineering, Chungnam National University

Jiro, Chikushi

Biotron Application Center, Kyushu University

他

https://doi.org/10.5109/20320

出版情報：九州大学大学院農学研究院紀要. 56 (2)，pp.271-277，2011-09. Faculty of Agriculture， Kyushu University

バージョン：

権利関係: 


\title{
The Effects of Noise and Vibration Generated by Mechanized Equipment in Laying Hen Houses on Productivity
}

\author{
Taek-Keun OH ${ }^{1 \dagger}$, Seung-Joo LEE $^{2 \dagger}$, Dong-Il CHANG ${ }^{2}$, \\ Hong-Hee CHANG ${ }^{3 *}$ and Jiro CHIKUSHI ${ }^{1}$
}

\author{
Biotron Application Center, Kyushu University, 6-10-1, Hakozaki, \\ Higashi-ku, Fukuoka 812-8581, Japan \\ (Received April 28, 2011 and accepted May 9, 2011)
}

\begin{abstract}
This study was carried out to investigate the effects of noise $(\mathrm{N})$ and vibration $(\mathrm{V})$ generated by mechanized equipment in laying hen houses on productivity of laying hens. N/V were measured at designated location in cages and facilities, and analyzed, with and without operation of feed supplier system, belt conveyor of layer feces, automatic egg collecting system, and feed distribution system at 13 mechanically-ventilated layer farms. Based on the characteristics of the $\mathrm{N} / \mathrm{V}$, levels of $\mathrm{N} / \mathrm{V}$ were established for layer rearing experiments, and productivity was analyzed. Maximum and mean levels for noise were 90 and $69 \mathrm{~dB}$, and they were 2.856 and $0.956 \mathrm{~cm} \mathrm{~s}^{-1}$ for vibration, respectively. Results showed that most noise and vibration were produced by feed supplier and feed distribution systems. Noise seemed not to affect productivity of laying hen, while vibration at levels greater than $1.0 \mathrm{~cm} \mathrm{~s}^{-1}$ did.
\end{abstract}

\section{INTRODUCTION}

Recently, due to the development of the Korean economy, food consumption pattern has changed and egg consumption per capita has increased. Higher egg consumption necessitates increased number of laying hens. Due to the development of the industry and livestock technology, traditional small-scale facilities have changed to large-scale mechanized units (National Agricultural Products Quality Management Service in Republic of Korea, 2005). Typical mechanical equipment within a laying hen house are ventilation system, feed supplier system, belt conveyor of layer feces, automatic egg collection system and feed distribution system. The studies on noise and vibration (N/V), affecting livestock productivity directly or indirectly, have conducted by Baek et al. (2002), Campo et al. (2005), Graul et al. (1976), Kim et al. (2001), Lee et al. (2003), Lee et al. (2004), Stephen et al. (1998), Warris et al. (1997), Yun (1998) and etc. All the results referenced above, were related to the effects of intermittent noise and vibration generated outside the layer hen houses on the productivity of layers in the houses. Otherwise, studies on the effects of noise and vibration generated and repeated by the facilities and machinery within the layer hen houses on the productivity of layers are limited.

The objective of this study was to measure and analyze N/V in laying hen houses equipped with multi-tier vertical cages when mechanical systems were or were

1 Biotron Application Center, Kyushu University, 6-10-1, Hakozaki, Higashi-ku, Fukuoka 812-8581, Japan

2 Department of Biosystem Machinery Engineering, Chungnam National University, Daejeon 305-764, South Korea

3 Department of Animal Science - Institute of Agriculture \& Life Sciences, College of Agriculture and Life Sciences, Gyeongsang National University, Jinju 660-701, South Korea

These two authors contributed equally to this work and should be considered co-first authors.

* Corresponding author (E-mail: hhchang@gnu.ac.kr) not in operation. Specific objectives of the study were to: (1) characterize N/V data measured in laying hen houses and (2) investigate the effects of N/V on productivity of laying hens through rearing experiments.

\section{MATERIALS AND METHODS}

\section{Test farms and measurement conditions}

$\mathrm{N} / \mathrm{V}$ measurements were taken on a nation-wide scale at 13 laying hen farms, located all over the country, and were measured for a week each barns. The farms are equipped with multi-tier vertical cages so as to investigate the characteristics of housing equipment at various levels of N/V (ASTM D 3580-95, 1995; ASTM E 756-05, 2005; KS B 0714, 2001; KS B 0712, 2003; Dong et al., 2005). Table 1 shows the list of surveyed laying hen farms for the N/V measurements.

The laying hen breed used in the test farms was the

Table 1. List of the surveyed laying hen farms for noise and vibration measurement

\begin{tabular}{cccc}
\hline $\begin{array}{c}\text { Name } \\
\text { of } \\
\text { farm }\end{array}$ & $\begin{array}{c}\text { No. of layer } \\
\text { (thousand head) }\end{array}$ & $\begin{array}{c}\text { Years of } \\
\text { mechanization } \\
\text { (yr) }\end{array}$ & $\begin{array}{c}\text { Type of } \\
\text { feed } \\
\text { supplier }\end{array}$ \\
\hline SY & 50 & 7.0 & Hopper \\
SS & 70 & 10.0 & Hopper \\
KP & 50 & 3.0 & Hopper \\
TH & 35 & 10.0 & Hopper \\
HA & 18 & 13.0 & Hopper \\
HJ & 40 & 1.0 & Hopper \\
SJCS & 27 & 8.0 & Hopper \\
MJG & 65 & 8.0 & Hopper \\
KPA & 12 & 3.0 & Chain \\
YS & 70 & 1.0 & Chain \\
YACS & 20 & 10.0 & Chain \\
YCS & 39 & 0.4 & Hopper \\
DS & 40 & 1.0 & Hopper \\
\hline
\end{tabular}


Hi-Line Brown. Operating schedule of the mechanical facilities of the laying hen houses were: (1) mechanical ventilation system operated continuously for 24 hours, (2) feed conveyor operated for 30 minutes 6-8 times during the lighting period, (3) feed distributor operated for 10-15 minutes after every operation of the feed supplier system and (4) feces belt and egg collection systems operated for two hours every morning. Normal light period lasted from $05: 00$ to $21: 00 \mathrm{~h}$, resulting in total lighting of 16 hours per day. After turning off the light no mechanical systems operated apart from the ventilation system.

Feces belt and egg collection system were same type at all 13 farms while type of feed supplier (feed supplier and feed distribution system) were different as Table 1.

\section{Measurement and analysis of N/V data}

N/V data were measured for a week at each 13 mechanically-ventilated laying hen farm with and without operation (normal condition) of the systems. And the operating schedules of mechanical systems for them were investigated.

Noise measurement regarding the ventilation system was taken at $2 \mathrm{~m}$ from the back side of the laying hen house. And for normal operation and for all the other mechanical systems, the measurement locations were at $2 \mathrm{~m}$ from the front side of the laying hen house as shown in Fig. 1 and Fig. 2. For the noise measurement and analysis, AC output values by sound level meter (NL-20) were saved with an oscilloscope (TDS5052B) and Vrms data were transformed to $\mathrm{dB}$ data by the calibration equation of NL-20 (spectrum technique). Noise recording with Panasonic PV400 was taken at same location as noise measurement location for reproducing noise of productivity experiment. Data on N/V of ventilation system were not taken, because its N/V might not directly or indirectly affect production and egg quality of laying hens. For vibration measurement and analysis, Vrms signals from the B\&K noise/vibration meter (logger : 5935L; accelerometer : 752A12) were saved with an oscilloscope (TDS5052B) through amplification circuit of the vibration meter, then measured signals were transformed to data of $\mathrm{cm} \mathrm{s}^{-1}$. Data were characterized by FFT (Fast

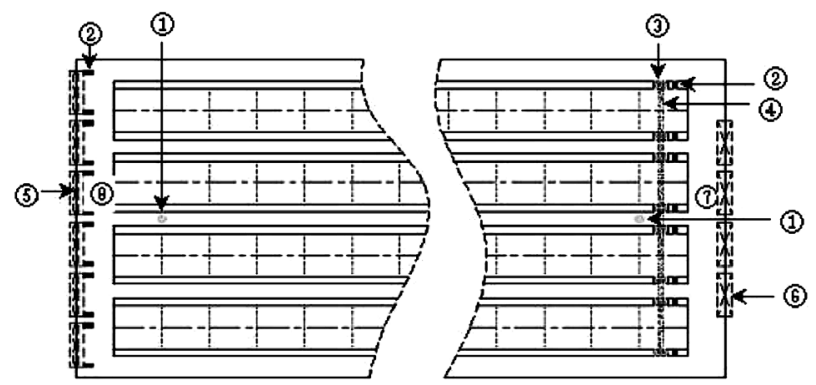

Fig. 1. Layout of noise/vibration measurement locations in laying hen houses (top view).

Conditions: (1)Noise measurement system (2)Vibration measurement system (3)Feed conveying system (4)Feed distribution system (5) Ventilation system (6) Air inlet (7)Front side 8)Back side.

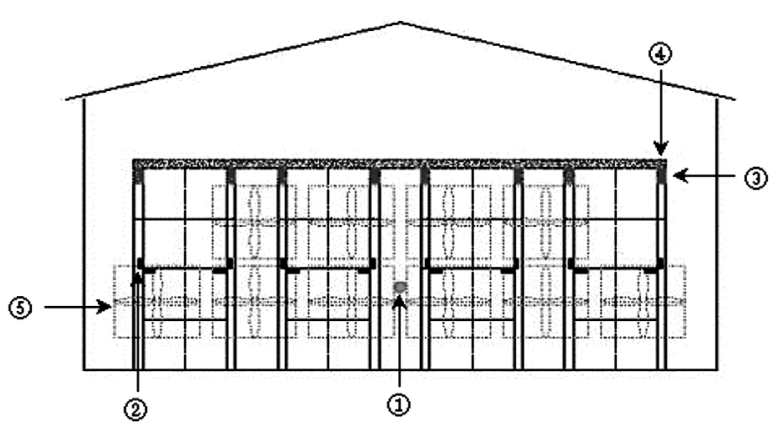

Fig. 2. Layout of noise/vibration measurement locations in laying hen house (front view).

Conditions: (1)Noise measurement system (2)Vibration measurement system (3)Feed conveying system (4)Feed distribution system (5)Ventilation system.

Fourier Transform) analysis technique.

Equipments for the N/V measurement and analysis are as follows:

- Sound level meter: RION NL-20 (RION Co. Ltd., Japan)

- Sound and video recording: Panasonic PV400 (Matsushita Inc., Japan)

- B\&K noise/vibration meter

- Logger: Dual Microphone Supply with LEMO Socket 5935L (B\&K Inc., Denmark, 2 channel)

- Accelerometer: General Purpose-Type 752A12 (B\&K Inc., Denmark)

- Oscilloscope: Tektronix TDS5052B (Tektronix Inc., U.S.A., 2 channel)

- $\quad$ Sound level meter (NL-20) and noise/vibration meter were calibrated by NC-73 (RION Co. Ltd., Japan)

- Portable Calibrators - Type 4294 (accelerometer calibrator, B\&K Inc., Denmark) and TDS5052B (probe calibrator included itself) before the test, respectively.

Sound and vibration levels were calibrated by NL-20, $\mathrm{B} \& \mathrm{~K}$ noise/vibration meter during productivity experiment with laying hens.

\section{Treatments for the rearing experiments}

The operating test schedule (Table 2) was established based on the general mechanical facilities operating schedule of 13 laying hen farms.

Nine treatments ( 3 levels of noise $\times 3$ levels of vibration) were designed and randomly allocated. Each treatment had six layers of Hi-Line Brown. Experiments were conducted in three replications and each replication took 30 days. Relocation of laying hens was carried out 10 days prior to beginning of each test and for the laying hens tested 10 days of stabilization period was used in order to reduce the effects of the relocation and previous test. The soundproofing walls were established in each cage for preventing to spread the noise.

It was assumed that the noise and vibration of the feed distribution system hardly affected in laying hens productivity of all cages, because it was located at front 
Table 2. Operating schedule of mechanical equipment for rearing tests

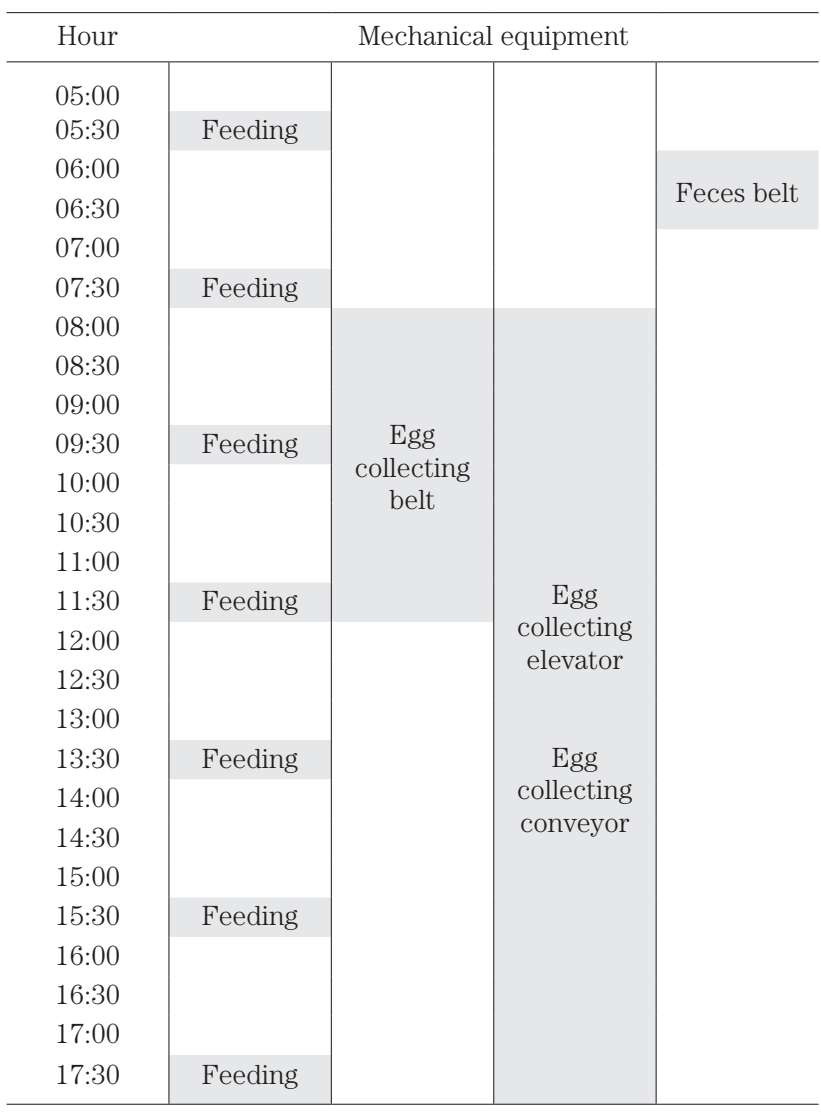

side in most 13 farms and length of cage was very long (about $100 \mathrm{~m}$ ) in laying hen house as above Fig. 1. Therefore, it was excluded during this experiment.

- The noise reproducing equipment and the vibration generator were operated for 10 minutes each on 05:30, 07:30, 09:30, 11:30, 13:30, 15:30 and 17:30, according to the operating schedule of the feed supplier system (Table 2). The selected three levels for noise were 70, 80 and $90 \mathrm{~dB}$ and for vibration were 0.3 (31.1), 1.0 (8.8) and $2.5 \mathrm{~cm} \mathrm{~s}^{-1}(1.7 \mathrm{~Hz})$ based on the result of this investigation. Noise was recorded by a camcorder in the laying hen house and was reproduced using a $\mathrm{PC}$, three amplifiers and nine speakers. In order to control the noise-reproducing equipment and the vibration generator, a program was developed using LabWindow/CVI 6.5 Package (National Instrument, USA). Levels of N/V were tested and adjusted with Sound level meter (RION NL-20, RION Co. Ltd., Japan) and B\&K noise/vibration meter (General Purpose-Type 752A12, B\&K Inc., Denmark). Fig. 3 shows a layout of the rearing experiment.

\section{Anaysis of N/ $\mathbf{V}$ effects on productivity of layinghens}

Lee et al. (2003) reported that the artificial noise affects productivity and stress of laying hens. So it was assumed that N/V affect to productivity and egg quality of laying hens in this study. Productivity of laying hens was analyzed in order to investigate the effects of $\mathrm{N} / \mathrm{V}$

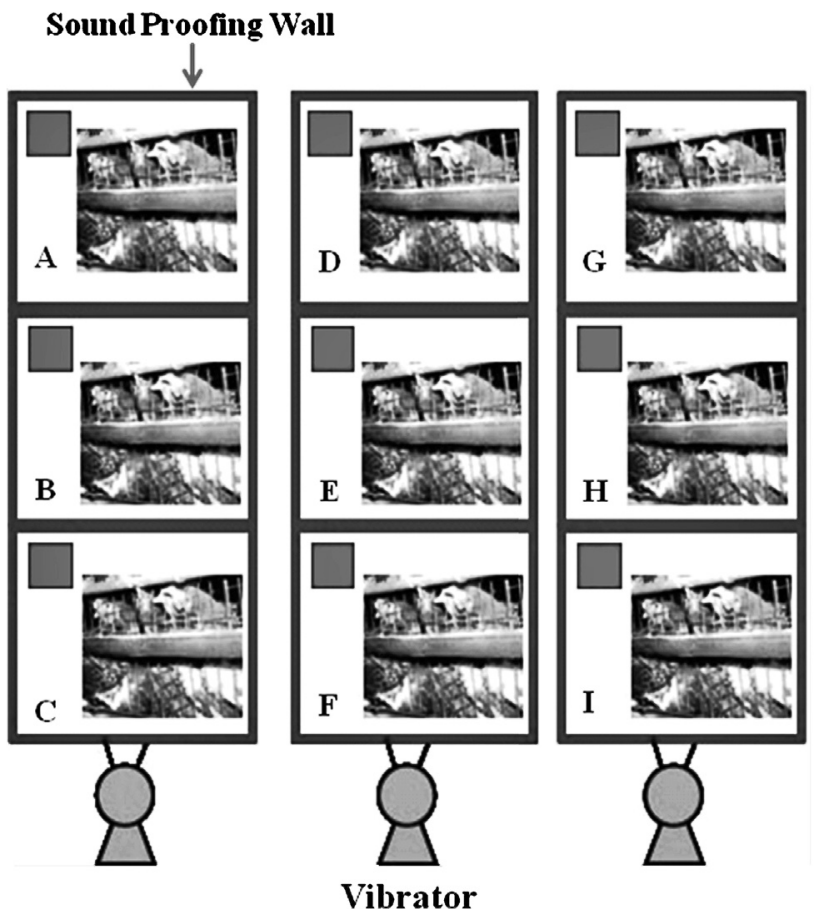

Fig. 3. Layout of the rearing experiment (A I : experimental treatment).

Conditions: A-70 dB \& $0.3 \mathrm{~cm} \mathrm{~s}^{-1}, \mathrm{~B}-80 \mathrm{~dB} \& 0.3 \mathrm{~cm} \mathrm{~s}^{-1}$, C-90 dB \& $0.3 \mathrm{~cm} \mathrm{~s}^{-1}, \mathrm{D}-70 \mathrm{~dB} \& 1.0 \mathrm{~cm} \mathrm{~s}^{-1}, \mathrm{E}-80 \mathrm{~dB}$ \& $1.0 \mathrm{~cm} \mathrm{~s}^{-1}, \mathrm{~F}-90 \mathrm{~dB} \& 1.0 \mathrm{~cm} \mathrm{~s}^{-1}, \mathrm{G}-70 \mathrm{~dB} \& 2.5 \mathrm{~cm} \mathrm{~s}^{-1}, \mathrm{H}-80$ $\mathrm{dB} \& 2.5 \mathrm{~cm} \mathrm{~s}^{-1}, \mathrm{I}-90 \mathrm{~dB} \& 2.5 \mathrm{~cm} \mathrm{~s}^{-1}$.

generated by the mechanical facilities within the laying hen houses. During the experimental period, at 10:00 AM every 10 days, quantity of feed intake, rate of eggs laying and quality of eggs (e.g. shell thickness, shell stiffness, weight of egg, HU (Haugh Unit) and xanthophylls) were measured. Shell thickness was measured by a shell thickness meter (Dial pipe gauge, Ozaki MFG Co. Ltd., Japan) and shell stiffness was measured by a shell stiffness meter (Egg shell force gauge model II, Robotmation Co. Ltd., Japan). Weight, HU, and xanthophylls were measured by Egg QCM+(Technical Services and Supplies, York Co. Ltd., England).

Measured data were analyzed using the SAS GLM procedure (SAS Inc., U.S.A., 1999) and significant differences were determined using the Duncan's multiple range test.

\section{RESULTS AND DISCUSSION}

Maximum levels of noise and vibration for each of the equipments within the laying hen houses were 82 (mean : 60) dB and 0.2027 (mean : 0.0985) $\mathrm{cm} \mathrm{s}^{-1}$ under normal condition, 90 (mean : 68) dB and 2.8560 (mean : $0.9563) \mathrm{cm} \mathrm{s}^{-1}$ for hopper type feed supplier system, 92 (mean : 69) dB and 1.0296 (mean : 0.2107) $\mathrm{cm} \mathrm{s}^{-1}$ for chain type feed supplier system, 88 (mean : 67) dB and 0.2387 (mean : 0.1523) $\mathrm{cm} \mathrm{s}^{-1}$ for belt conveyer for layer feces, 87 (mean : 67) dB and 0.1865 (mean : 0.0928) cm $\mathrm{s}^{-1}$ for automatic egg collection system and 90 (mean : 69) $\mathrm{dB}$ and 2.0222 (mean : 0.9214) $\mathrm{cm} \mathrm{s}^{-1}$ for feed distribution system, respectively. The levels of noise and 
Table 3. Results at 1st day of experiment

\begin{tabular}{|c|c|c|c|c|c|c|c|c|}
\hline \multicolumn{2}{|c|}{ Treatment } & \multirow{2}{*}{$\begin{array}{l}\text { Quantity of } \\
\text { feed intake } \\
\left(\mathrm{g} \mathrm{hen}^{-1} \text { day }^{-1}\right)\end{array}$} & \multirow{2}{*}{$\begin{array}{l}\text { Rates of } \\
\text { laying eggs } \\
(\%)\end{array}$} & \multirow[b]{2}{*}{$\begin{array}{l}\text { Weight of egg } \\
\text { (g) }\end{array}$} & \multirow{2}{*}{$\begin{array}{c}\text { Shell } \\
\text { thickness } \\
(\mu \mathrm{m})\end{array}$} & \multirow[b]{2}{*}{$\begin{array}{l}\text { Shell stiffness } \\
\left(\mathrm{kg} \mathrm{cm}^{2-1}\right)\end{array}$} & \multirow[b]{2}{*}{$\mathrm{HU}$} & \multirow[b]{2}{*}{ Xanthophylls } \\
\hline $\begin{array}{l}\text { Vibration } \\
\left(\mathrm{cm} \mathrm{s}^{-1}\right)\end{array}$ & $\begin{array}{l}\text { Noise } \\
(\mathrm{dB})\end{array}$ & & & & & & & \\
\hline 0.3 & 70 & 165.8 & 94.4 & 58.3 & 541.2 & 4.7 & 91.0 & 12.1 \\
\hline 0.3 & 80 & 166.7 & 94.4 & 57.5 & 539.1 & 4.5 & 92.7 & 12.4 \\
\hline 0.3 & 90 & 172.2 & 94.4 & 58.5 & 539.0 & 4.8 & 93.5 & 12.1 \\
\hline 1.0 & 70 & 163.8 & 94.4 & 55.9 & 540.3 & 4.3 & 92.9 & 12.4 \\
\hline 1.0 & 80 & 164.5 & 94.4 & 57.8 & 540.0 & 4.7 & 94.8 & 12.2 \\
\hline 1.0 & 90 & 160.0 & 94.4 & 58.3 & 539.3 & 4.7 & 92.1 & 12.2 \\
\hline 2.5 & 70 & 167.5 & 94.4 & 58.0 & 540.2 & 4.3 & 92.4 & 12.5 \\
\hline 2.5 & 80 & 167.2 & 89.9 & 57.3 & 539.0 & 4.9 & 92.1 & 12.3 \\
\hline 2.5 & 90 & 166.7 & 88.9 & 57.8 & 539.1 & 4.5 & 92.8 & 12.0 \\
\hline \multicolumn{2}{|c|}{ SEM } & 3.07 & 1.61 & 0.28 & 0.00 & 0.07 & 0.45 & 0.05 \\
\hline \multicolumn{2}{|c|}{ Items } & $\begin{array}{l}\text { Degree of } \\
\text { freedom }\end{array}$ & \multicolumn{6}{|c|}{ Significance probability } \\
\hline Treatment & 8 & 0.9991 & 0.9882 & 0.5321 & 0.5057 & 0.3878 & 0.7596 & 0.4941 \\
\hline Vibration & 2 & 0.8084 & 0.6480 & 0.5168 & 0.7406 & 0.7591 & 0.6445 & 0.7049 \\
\hline Noise & 2 & 0.9976 & 0.8954 & 0.4532 & 0.1683 & 0.2146 & 0.6220 & 0.1877 \\
\hline $\begin{array}{c}\text { Vibration \& } \\
\text { noise }\end{array}$ & 4 & 0.9888 & 0.9770 & 0.3902 & 0.5411 & 0.3037 & 0.5383 & 0.5035 \\
\hline \multicolumn{9}{|c|}{ Main effect } \\
\hline \multirow{3}{*}{$\begin{array}{l}\text { Vibration } \\
\left(\mathrm{cm} \mathrm{s}^{-1}\right)\end{array}$} & 0.3 & 168.2 & 94.4 & 58.1 & 539.6 & 4.7 & 92.3 & 12.2 \\
\hline & 1.0 & 162.7 & 94.4 & 57.3 & 539.5 & 4.6 & 93.3 & 12.3 \\
\hline & 2.5 & 167.1 & 90.7 & 57.7 & 539.2 & 4.6 & 92.4 & 12.3 \\
\hline \multirow{3}{*}{$\begin{array}{l}\text { Noise } \\
(\mathrm{dB})\end{array}$} & 70 & 165.7 & 94.4 & 57.4 & 539.9 & 4.5 & 92.1 & 12.3 \\
\hline & 80 & 166.1 & 92.6 & 57.5 & 539.2 & 4.7 & 93.1 & 12.3 \\
\hline & 90 & 166.3 & 92.6 & 58.2 & 539.2 & 4.7 & 92.8 & 12.1 \\
\hline
\end{tabular}

* SEM : Standard Error of Means

Table 4. Results at 10th day of experiment

\begin{tabular}{|c|c|c|c|c|c|c|c|c|}
\hline \multicolumn{2}{|c|}{ Treatment } & \multirow{2}{*}{$\begin{array}{l}\text { Quantity of } \\
\text { feed intake } \\
\left(\mathrm{g} \mathrm{hen}^{-1} \text { day }^{-1}\right)\end{array}$} & \multirow{2}{*}{$\begin{array}{l}\text { Rates of } \\
\text { laying eggs } \\
(\%)\end{array}$} & \multirow[b]{2}{*}{$\begin{array}{l}\text { Weight of egg } \\
\text { (g) }\end{array}$} & \multirow{2}{*}{$\begin{array}{c}\text { Shell } \\
\text { thickness } \\
(\mu \mathrm{m})\end{array}$} & \multirow[b]{2}{*}{$\begin{array}{l}\text { Shell stiffness } \\
\left(\mathrm{kg} \mathrm{cm}^{2-1}\right)\end{array}$} & \multirow[b]{2}{*}{$\mathrm{HU}$} & \multirow[b]{2}{*}{ Xanthophylls } \\
\hline $\begin{array}{l}\text { Vibration } \\
\left(\mathrm{cm} \mathrm{s}^{-1}\right)\end{array}$ & $\begin{array}{l}\text { Noise } \\
(\mathrm{dB})\end{array}$ & & & & & & & \\
\hline 0.3 & 70 & 167.2 & $94.4^{\mathrm{a}}$ & 57.6 & 538.2 & 4.2 & $96.8^{\mathrm{C}}$ & 11.9 \\
\hline 0.3 & 80 & 168.0 & $94.4^{\mathrm{a}}$ & 57.6 & 538.4 & 3.9 & $96.7^{\mathrm{C}}$ & 12.3 \\
\hline 0.3 & 90 & 171.4 & $94.4^{\mathrm{a}}$ & 58.7 & 539.3 & 3.9 & $103.5^{\mathrm{A}}$ & 11.9 \\
\hline 1.0 & 70 & 161.9 & $83.3^{\mathrm{ab}}$ & 57.5 & 537.6 & 4.0 & $97.9^{\mathrm{BC}}$ & 12.3 \\
\hline 1.0 & 80 & 163.9 & $77.8^{\mathrm{ab}}$ & 56.8 & 537.5 & 4.2 & $96.5^{\mathrm{C}}$ & 12.1 \\
\hline 1.0 & 90 & 160.5 & $78.8^{\mathrm{ab}}$ & 56.8 & 538.2 & 4.1 & $103.5^{\mathrm{A}}$ & 12.1 \\
\hline 2.5 & 70 & 163.3 & $72.2^{\mathrm{b}}$ & 58.3 & 537.4 & 3.7 & $98.6^{\mathrm{BC}}$ & 12.4 \\
\hline 2.5 & 80 & 165.3 & $77.8^{\mathrm{ab}}$ & 58.7 & 537.3 & 4.0 & $101.4^{\mathrm{AB}}$ & 12.2 \\
\hline 2.5 & 90 & 166.4 & $77.8^{\mathrm{ab}}$ & 58.4 & 538.1 & 4.4 & $101.6^{\mathrm{AB}}$ & 12.2 \\
\hline \multicolumn{2}{|c|}{ SEM } & 1.21 & 2.18 & 0.53 & 0.00 & 0.08 & 0.48 & 0.06 \\
\hline \multicolumn{2}{|c|}{ Items } & $\begin{array}{l}\text { Degree of } \\
\text { freedom }\end{array}$ & \multicolumn{6}{|c|}{ Significance probability } \\
\hline Treatment & 8 & 0.6165 & 0.2633 & 0.8795 & 0.4418 & 0.6653 & 0.0001 & 0.3185 \\
\hline Vibration & 2 & 0.1150 & 0.0177 & 0.2827 & 0.1487 & 0.8918 & 0.2526 & 0.1789 \\
\hline Noise & 2 & 0.7953 & 0.9199 & 0.9528 & 0.3482 & 0.7830 & 0.0001 & 0.5333 \\
\hline $\begin{array}{c}\text { Vibration \& } \\
\text { noise }\end{array}$ & 4 & 0.9086 & 0.9306 & 0.8997 & 0.7399 & 0.2817 & 0.0774 & 0.3298 \\
\hline \multicolumn{9}{|c|}{ Main effect } \\
\hline \multirow{3}{*}{$\begin{array}{l}\text { Vibration } \\
\left(\mathrm{cm} \mathrm{s}^{-1}\right)\end{array}$} & 0.3 & 168.9 & $92.6^{\mathrm{A}}$ & 57.9 & 538.2 & 4.0 & 98.9 & 12.0 \\
\hline & 1.0 & 162.1 & $83.3^{\mathrm{AB}}$ & 57.0 & 537.2 & 4.1 & 99.3 & 12.2 \\
\hline & 2.5 & 165.0 & $75.9^{\mathrm{B}}$ & 58.5 & 537.5 & 4.0 & 100.6 & 12.3 \\
\hline \multirow{3}{*}{$\begin{array}{l}\text { Noise } \\
(\mathrm{dB})\end{array}$} & 70 & 164.1 & 83.3 & 57.8 & 537.3 & 4.0 & $97.7^{\mathrm{B}}$ & 12.2 \\
\hline & 80 & 165.7 & 85.2 & 57.7 & 537.5 & 4.1 & $98.1^{\mathrm{B}}$ & 12.2 \\
\hline & 90 & 166.1 & 83.3 & 57.9 & 538.1 & 4.1 & $102.9^{\mathrm{A}}$ & 12.1 \\
\hline
\end{tabular}

${ }^{a, b, c}$ : Means with the different superscripts in the same column differ significantly $(p<0.05)$.

${ }^{A, B, C}$ : Means with the different superscripts in the same column differ significantly $(p<0.01)$.

* SEM : Standard Error of Means 
Table 5. Results at 20th day of experiment

\begin{tabular}{|c|c|c|c|c|c|c|c|c|}
\hline \multicolumn{2}{|c|}{ Treatment } & \multirow{2}{*}{$\begin{array}{l}\text { Quantity of } \\
\text { feed intake } \\
\left(\mathrm{g} \mathrm{hen}^{-1} \text { day }^{-1}\right)\end{array}$} & \multirow{2}{*}{$\begin{array}{l}\text { Rates of } \\
\text { laying eggs } \\
(\%)\end{array}$} & \multirow[b]{2}{*}{$\begin{array}{l}\text { Weight of egg } \\
\text { (g) }\end{array}$} & \multirow{2}{*}{$\begin{array}{c}\text { Shell } \\
\text { thickness } \\
(\mu \mathrm{m})\end{array}$} & \multirow[b]{2}{*}{$\begin{array}{l}\text { Shell stiffness } \\
\left(\mathrm{kg} \mathrm{cm}^{2-1}\right)\end{array}$} & \multirow[b]{2}{*}{$\mathrm{HU}$} & \multirow[b]{2}{*}{ Xanthophylls } \\
\hline $\begin{array}{l}\text { Vibration } \\
\left(\mathrm{cm} \mathrm{s}^{-1}\right)\end{array}$ & $\begin{array}{l}\text { Noise } \\
(\mathrm{dB})\end{array}$ & & & & & & & \\
\hline 0.3 & 70 & 172.7 & $100.0^{\mathrm{A}}$ & 59.5 & 537.6 & 4.1 & 96.1 & 12.4 \\
\hline 0.3 & 80 & 175.7 & $94.4^{\mathrm{AB}}$ & 58.7 & 536.7 & 4.2 & 96.8 & 12.5 \\
\hline 0.3 & 90 & 168.0 & $94.4^{\mathrm{AB}}$ & 62.7 & 535.9 & 4.3 & 99.5 & 12.5 \\
\hline 1.0 & 70 & 161.7 & $77.8^{\mathrm{C}}$ & 59.8 & 535.5 & 4.0 & 96.8 & 12.6 \\
\hline 1.0 & 80 & 161.0 & $88.9^{\mathrm{ABC}}$ & 59.9 & 536.4 & 4.2 & 95.0 & 12.4 \\
\hline 1.0 & 90 & 165.7 & $88.9^{\mathrm{ABC}}$ & 59.5 & 537.3 & 4.3 & 96.6 & 12.6 \\
\hline 2.5 & 70 & 158.3 & $83.3^{\mathrm{BC}}$ & 61.2 & 536.5 & 4.0 & 96.8 & 12.4 \\
\hline 2.5 & 80 & 170.0 & $77.8^{\mathrm{C}}$ & 61.3 & 535.9 & 4.1 & 98.5 & 12.6 \\
\hline 2.5 & 90 & 159.3 & $77.8^{\mathrm{C}}$ & 60.3 & 535.5 & 4.0 & 97.6 & 12.4 \\
\hline \multicolumn{2}{|c|}{ SEM } & 2.31 & 2.05 & 0.38 & 0.00 & 0.06 & 0.38 & 0.05 \\
\hline \multicolumn{2}{|c|}{ Items } & $\begin{array}{l}\text { Degree of } \\
\text { freedom }\end{array}$ & \multicolumn{6}{|c|}{ Significance probability } \\
\hline Treatment & 8 & 0.6673 & 0.0290 & 0.2366 & 0.1307 & 0.8505 & 0.2006 & 0.9296 \\
\hline Vibration & 2 & 0.2105 & 0.0019 & 0.3433 & 0.1346 & 0.4975 & 0.2070 & 0.7200 \\
\hline Noise & 2 & 0.6725 & 1.0000 & 0.4305 & 0.6929 & 0.5045 & 0.2466 & 0.9455 \\
\hline $\begin{array}{c}\text { Vibration \& } \\
\text { noise }\end{array}$ & 4 & 0.8068 & 0.3129 & 0.1569 & 0.0985 & 0.8662 & 0.2722 & 0.6867 \\
\hline \multicolumn{9}{|c|}{ Main effect } \\
\hline \multirow{3}{*}{$\begin{array}{l}\text { Vibration } \\
\left(\mathrm{cm} \mathrm{s}^{-1}\right)\end{array}$} & 0.3 & 172.1 & $96.3^{\mathrm{A}}$ & 60.3 & 536.4 & 4.2 & 97.4 & 12.4 \\
\hline & 1.0 & 162.8 & $85.2^{\mathrm{B}}$ & 59.7 & 536.2 & 4.2 & 96.1 & 12.5 \\
\hline & 2.5 & 162.6 & $79.6^{\mathrm{B}}$ & 61.1 & 535.2 & 4.0 & 97.6 & 12.5 \\
\hline \multirow{3}{*}{$\begin{array}{l}\text { Noise } \\
(\mathrm{dB})\end{array}$} & 70 & 164.2 & 87.0 & 60.1 & 536.3 & 4.0 & 96.5 & 12.5 \\
\hline & 80 & 168.9 & 87.0 & 59.9 & 535.7 & 4.2 & 96.7 & 12.5 \\
\hline & 90 & 164.3 & 87.0 & 61.0 & 535.9 & 4.2 & 98.0 & 12.5 \\
\hline
\end{tabular}

* SEM and ${ }^{\mathrm{A}, \mathrm{B}, \mathrm{C}}$ : See Table 4.

Table 6. Results at 30th day of experiment

\begin{tabular}{|c|c|c|c|c|c|c|c|c|}
\hline \multicolumn{2}{|c|}{ Treatment } & \multirow{2}{*}{$\begin{array}{l}\text { Quantity of } \\
\text { feed intake } \\
\left(\mathrm{g} \mathrm{hen}^{-1} \text { day }^{-1}\right)\end{array}$} & \multirow{2}{*}{$\begin{array}{l}\text { Rates of } \\
\text { laying eggs } \\
(\%)\end{array}$} & \multirow[b]{2}{*}{$\begin{array}{l}\text { Weight of egg } \\
\text { (g) }\end{array}$} & \multirow{2}{*}{$\begin{array}{c}\text { Shell } \\
\text { thickness } \\
(\mu \mathrm{m})\end{array}$} & \multirow[b]{2}{*}{$\begin{array}{l}\text { Shell stiffness } \\
\left(\mathrm{kg} \mathrm{cm}^{2-1}\right)\end{array}$} & \multirow[b]{2}{*}{$\mathrm{HU}$} & \multirow[b]{2}{*}{ Xanthophylls } \\
\hline $\begin{array}{l}\text { Vibration } \\
\left(\mathrm{cm} \mathrm{s}^{-1}\right)\end{array}$ & $\begin{array}{l}\text { Noise } \\
(\mathrm{dB})\end{array}$ & & & & & & & \\
\hline 0.3 & 70 & 166.7 & $94.4^{\mathrm{A}}$ & 61.9 & 534.3 & 3.9 & 91.9 & 12.6 \\
\hline 0.3 & 80 & 168.0 & $94.4^{\mathrm{A}}$ & 62.0 & 533.9 & 3.9 & 91.8 & 12.6 \\
\hline 0.3 & 90 & 164.3 & $94.4^{\mathrm{A}}$ & 62.4 & 434.3 & 4.0 & 93.1 & 12.5 \\
\hline 1.0 & 70 & 174.3 & $83.0^{\mathrm{AB}}$ & 62.1 & 533.7 & 4.2 & 91.5 & 12.9 \\
\hline 1.0 & 80 & 170.3 & $83.3^{\mathrm{AB}}$ & 63.8 & 534.5 & 3.9 & 92.3 & 12.7 \\
\hline 1.0 & 90 & 165.0 & $88.9^{\mathrm{AB}}$ & 64.0 & 533.6 & 3.7 & 91.6 & 12.6 \\
\hline 2.5 & 70 & 167.8 & $72.2^{\mathrm{B}}$ & 62.5 & 534.2 & 3.9 & 91.4 & 12.6 \\
\hline 2.5 & 80 & 171.7 & $72.2^{\mathrm{B}}$ & 61.3 & 535.0 & 4.1 & 93.5 & 12.5 \\
\hline 2.5 & 90 & 170.3 & $77.8^{\mathrm{AB}}$ & 62.7 & 535.1 & 4.2 & 92.2 & 12.6 \\
\hline \multicolumn{2}{|c|}{ SEM } & 1.54 & 2.34 & 0.34 & 0.00 & 0.06 & 0.29 & 0.06 \\
\hline \multicolumn{2}{|c|}{ Items } & $\begin{array}{l}\text { Degree of } \\
\text { freedom }\end{array}$ & \multicolumn{6}{|c|}{ Significance probability } \\
\hline Treatment & 8 & 0.9139 & 0.0566 & 0.6917 & 0.8494 & 0.6262 & 0.7359 & 0.9283 \\
\hline Vibration & 2 & 0.6299 & 0.0019 & 0.2951 & 0.1906 & 0.4289 & 0.6920 & 0.4848 \\
\hline Noise & 2 & 0.6789 & 0.6771 & 0.5505 & 0.9580 & 0.9169 & 0.5027 & 0.6215 \\
\hline $\begin{array}{c}\text { Vibration \& } \\
\text { noise }\end{array}$ & 4 & 0.8489 & 0.9812 & 0.7485 & 0.9616 & 0.3699 & 0.5493 & 0.9571 \\
\hline \multicolumn{9}{|c|}{ Main effect } \\
\hline \multirow{3}{*}{$\begin{array}{l}\text { Vibration } \\
\left(\mathrm{cm} \mathrm{s}^{-1}\right)\end{array}$} & 0.3 & 166.3 & $94.4^{\mathrm{A}}$ & 62.1 & 533.6 & 3.9 & 92.3 & 12.6 \\
\hline & 1.0 & 169.8 & $85.2^{\mathrm{B}}$ & 63.3 & 533.4 & 3.9 & 91.8 & 12.7 \\
\hline & 2.5 & 169.9 & $74.1^{\mathrm{C}}$ & 62.2 & 534.7 & 4.1 & 92.4 & 12.6 \\
\hline \multirow{3}{*}{$\begin{array}{l}\text { Noise } \\
(\mathrm{dB})\end{array}$} & 70 & 169.4 & 83.3 & 62.1 & 533.8 & 4.0 & 91.6 & 12.7 \\
\hline & 80 & 170.0 & 83.3 & 63.3 & 533.8 & 3.9 & 92.4 & 12.6 \\
\hline & 90 & 166.6 & 87.0 & 62.2 & 534.0 & 4.0 & 92.3 & 12.6 \\
\hline
\end{tabular}

* SEM and ${ }^{\mathrm{A}, \mathrm{B}, \mathrm{C}}$ : See Table 4. 
vibration of feed supplier system and the feed distribution system were higher than $50 \mathrm{~dB}$ and $0.3 \mathrm{~cm} \mathrm{~s}^{-1}$ of intermittent construction N/V which may induce decrease of productivity in laying hens by KME (Korean Ministry of Environment, 2002). Repeated noise does not affect production (rates of laying eggs and weight of egg) and egg quality (shell thickness, shell stiffness, HU and Xanthophylls) in laying hens (Graul et al., 1976; Lee et al., 2004), but vibration may affect egg production of laying hens by the result of this study. Therefore, chain type feed supplier system with lower vibration levels is suitable than hopper type with higher in laying hen house.

Results of rearing experiments are summarized in Table 3, Table 4, Table 5 and Table 6. During the experimental period, the noise and vibration levels seem not affect quantity of feed intake, weight of egg, shell thickness, shell stiffness, and xanthophylls ( $p>0.05$ ). Furthermore, HU was affected by N/V on the 10th day $(p<0.01)$, but not on the 20th day and the 30th day of experiment $(p>0.05)$. Finally, rates of laying eggs were not affected by noise $(p>0.05)$, but they were affected by vibration $(\mathrm{p}<0.01)$. In other words, rates of laying eggs (92.6 to $96.3 \%$ ) at vibration velocity of $0.3 \mathrm{~cm} \mathrm{~s}^{-1}$ was significantly $(p<0.01)$ greater than those $(74.1$ to $85.2 \%$ ) at vibration velocities of 1.0 and $2.5 \mathrm{~cm} \mathrm{~s}^{-1}$. Based on the level of vibration affecting productivity of laying hens, a design guideline for vibration protection could be proposed and it would be applied for the optimum design condition of vibration for the mechanical systems within laying hen houses.

When livestock are exported to intermittent loud noise, heart rate, breathing rate and secretion of stress hormone was increased while rate of laying eggs and growth rate were decreased. And mortality, abortion and stillbirth may happen (Baek et al., 2002; Kim et al., 2001; Yun, 1998). However, because laying hens exposed to repeated artificial construction noise stress back to initial status over seven days by Graul et al. (1976) and Lee et al. (2004), noise did not affect production and egg quality in laying hens. Noise from indoor laying hen house is repeated noise. These previous results accord with the findings of this study. Vibration from construction affects production of laying hens by KME (Korean Ministry of Environment, 2002). There are no findings of vibration effect on egg quality in laying hens until these days.

Considering these previous results and our results, repeated vibration affects production of laying hens while repeated noise does not affect it.

\section{CONCLUSIONS}

This study was carried out to investigate the effects of N/V generated by the mechanical systems (ventilation fans, feed supplier system, belt conveyor of layer feces, automatic egg collection system and feed distribution system) in laying hen houses on productivity of laying hens. $\mathrm{N} / \mathrm{V}$ were measured and analyzed at designated points of cages and facilities. Maximum levels of noise for each of the equipment were $82 \mathrm{~dB}$ (normal condition) to $92 \mathrm{~dB}$ (chain type feed supplier system) and vibration were
$0.2027 \mathrm{~cm} \mathrm{~s}^{-1}$ (normal condition) to $2.8560 \mathrm{~cm} \mathrm{~s}^{-1}$ (hopper type feed supplier system). Based on the characteristics of N/V various levels, N/V levels were established for rearing experiments (noise : 70, 80 and $90 \mathrm{~dB}$; vibration : $0.3,1.0$, and $2.5 \mathrm{~cm} \mathrm{~s}^{-1}$ ). Nine treatments (3 levels of noise $\times 3$ levels of vibration) were selected and randomly allocated. Then tests were conducted, and productivity (feed intake and egg production) and egg quality (shell thickness, shell stiffness, weight of egg, Haugh Unit and xanthophylls) were analyzed. Major findings were: (1) Noise seemed not to affecting productivity of laying hens while significantly impacted HU on Day 10, (2) Vibration only affected egg production at levels greater than $1.0 \mathrm{~cm} \mathrm{~s}^{-1}$, and others were not affected by vibration. Based on the level of vibration affecting egg production, a design guideline for vibration protection could be proposed and it would be applied for the optimum design condition of vibration for the mechanical systems in laying hen houses.

\section{REFERENCES}

American Society for Testing Materials 1995 In "Standard test methods for vibration (vertical linear motion) test of products. ASTM Standards D 3580-95"

American Society for Testing Materials 2005 In "Standard test method for measuring vibration - Damping properties of materials. ASTM Standards E 756-05"

Baek, Y. J., J. S. Choi, K. J. Kim and D. M. Bae 2002 A case study on the analysis of the noise damage characterization of livestock. Proceedings of the Korean Society for Noise and Vibration Engineering 2002 Fall Conference, 2002(2): $755-761$

Campo, J. L., M. G. Gil and S. G. Davila 2005 Effects of specific noise and music stimuli on stress and fear levels of laying hens several breeds. Applied Animal Behaviour Science, 91: $75-84$

Dong, J. Y. and S. K. Lee 2005 Development of management system for measurement and characteristic analysis, evaluation of environmental noise. J. of Korean Society for Noise and Vibration Engineering, $\mathbf{1 5}$ (3): 306-312

Graul, C., V. Wildenhan, L. Lyhs and W. Lohse 1976 Effect of noise on the physiological functions in fowl. 2. Adaptation to noise of white leghorns and broilers. Archiv für experimentelle Veterinärmedizin, 30(5): 643-650

Hong, J. C., K. S. Sun and Y. Y. Kim 2005 Adaptive short-time fourier transform for guided-wave analysis. J. of Korean Society for Noise and Vibration Engineering, 15(3): 266271. Available at: www.ksnve.or.kr/papers.htm

Kim, J. S., K. S. Kwack, B. Y. Lee, J. Y. Park, H. J. Lee and G. C. Jeong 2001 Noise and Vibration of Construction

Korean Ministry of Environment 2002 Study on Evaluation Method of Livestock Injury by Noise and Vibration. Report of Korean Ministry of Environment

Korean Standards Association 2001 In "Mechanical vibration and shock - Mechanical mounting of accelerometer. Korean Industrial Standards B 0714"

Korean Standards Association 2003 In "Mechanical vibration and shock-Vibration of buildings - Guidelines for the measurement of vibrations and evaluation of their effects on buildings. Korean Industrial Standards B 0712"

Lee, D. S., H. S. Kang, H. C. Choi, D. J. Kwon, D. G. Kim and S. I. Jeong 2004 Effect of noise on production of livestock. Korean Rural Development Administration Research Report

Lee, H. M., S. Kim and J. G. Lee 2003 Investigation of abnormal eggs and cortisol stress hormone in laying hens due to the artificial noise. J. of Korean Society of Environmental Engineering, 25(7): 860-865 
National Agricultural Products Quality Management Service in Republic of Korea 2005 The Korean Agriculture Statistics - The part of Livestock

Stephen, J. G. Hall and R. Harry Bradshaw 1998 Welfare aspect of transport by road of sheep and pigs. J. of Applied Animal Welfare Science, 1(3): 235-254

Warriss, P. D., S. N. Brown, T. G. Kwonles, J. E. Edwards and J. A.
Duggan 1997 Potential effect of vibration during transport on glycogen reserves in broiler chicken. The Veterinary Journal, 153(2): 215-219

Yun, H. J 1998 Relationship between human and livestock injury by construction noise. Proceedings of the Korean Society for Noise and Vibration Engineering 1998 Spring Conference, 1998(2): 710-721 